\title{
Therapy for idiopathic pulmonary fibrosis: lessons from pooled data analyses
}

\author{
Lauren K. Troy ${ }^{1,2}$ and Tamera J. Corte ${ }^{1,2}$
}

Affiliations: ${ }^{1}$ Dept of Respiratory Medicine, Royal Prince Alfred Hospital, Sydney, Australia. ${ }^{2}$ Sydney Medical School, University of Sydney, Sydney, Australia.

Correspondence: Tamera J. Corte, Dept of Respiratory and Sleep Medicine, Royal Prince Alfred Hospital, Missenden Road, Camperdown, Sydney, NSW 2050, Australia. E-mail: tameracorteamac.com

○ $@$ ERSpublications

The pooled analysis of three large phase 3 trials sheds further light on the role of antifibrotic therapy in IPF http://ow.ly/UNTWQ

In 2014, two landmark clinical trials (ASCEND and INPULSIS) were published demonstrating, for the first time, a treatment benefit for patients with idiopathic pulmonary fibrosis (IPF). Both pirfenidone and nintedanib were shown to have a clear therapeutic benefit in slowing the relentless disease progression evident in IPF patients. The results of these studies have had a major impact upon management of IPF patients worldwide, with subsequent changes made to international guidelines, which now recommend in favour of antifibrotic therapy. However, this is not the whole story: there are many unanswered questions with regard to the real-world treatment benefit and use of antifibrotic therapy. In this issue of the European Respiratory Journal, NoBLE et al. [1] present the pooled data of the three multinational, phase 3, placebo-controlled trials of pirfenidone in IPF patients, the ASCEND, and CAPACITY 004 and 006 studies. Examining the combined patient population reveals important insights about specific subgroups along with broader inferences that are only possible through pooled analysis.

The history of clinical trials of pirfenidone in IPF patients is intriguing: 2011 saw the publication of two concurrent phase 3 trials (CAPACITY 004 and 006) [2]. These followed on from the promising findings of pre-clinical [3-5] and phase 2 studies [6,7], and a large phase 3 trial conducted in Japanese patients [8]. Unexpectedly, the results of the CAPACITY studies were disparate: CAPACITY 004 showed a significant reduction in forced vital capacity (FVC) decline and an improvement in progression-free survival at 72 weeks [2]; CAPACITY 006 was a negative study, with failure to meet either of these end-points [2].

Why were the CAPACITY 004 and 006 results conflicting when the study populations were ostensibly the same? Subtle differences, in fact, did exist between the patient cohorts [2]. Interestingly, a difference in the change in FVC in the placebo arm was evident between the two studies. Further scrutiny revealed a greater degree of airflow limitation, as indicated by a lower spirometric ratio, in 006 than in 004 . Perhaps this could explain the observed variation in physiological behaviour.

Pirfenidone was subsequently approved for use across Europe based on its treatment effect demonstrated in the pooled analysis of the CAPACITY trials and previous data from Japanese studies [7-9]. However, in other countries, including the USA, regulatory bodies required further evidence of efficacy. Lessons were learnt and enabled enrichment of the ASCEND study cohort (a phase 3 study of 52 weeks duration) for patients at risk of disease progression, a strategy that has been used successfully in the study of agents for other diseases [10]. A major change was the centralisation of review of IPF diagnosis. The primary end-point remained "change in percentage predicted FVC or death", with the magnitude of the treatment effect measured categorically as the proportion of subjects with $\geqslant 10 \%$ fall in FVC, based on the

Received: Oct 092015 | Accepted after revision: Nov 152015

Conflict of interest: Disclosures can be found alongside the online version of this article at erj.ersjournals.com

Copyright @ERS 2016 
CAPACITY findings. At study completion, the primary outcome was positive, with a relative reduction of $47.9 \%$ in the proportion reaching the composite end-point of $\geqslant 10 \%$ fall in FVC or death [11].

In a pre-specified pooled analysis across the combined ASCEND and CAPACITY populations, pirfenidone was shown to reduce the risk of death from any cause at 1 year by $48 \%$ and death due to IPF by $68 \%$, compared with placebo [11]. These findings were published simultaneously with the combined results of the INPULSIS 1 and 2 parallel phase 3 studies in the triple kinase inhibitor nintedanib [12]. These, too, were positive studies, demonstrating a slowing in the decline of FVC for those on active therapy. The landmark publications of the two antifibrotic agents were quickly followed by US Food and Drug Administration approval, and the release of updated IPF therapeutic guidelines [13].

In their analysis of ASCEND and CAPACITY 004 and 006 study data, Noble et al. [1] report the pooled efficacy and side-effects of pirfenidone in IPF patients. These trials combined included $>1200$ patients, half each receiving pirfenidone or placebo $[2,11]$. Pre-specified, categorical end-points, including the percentage of patients with a $10 \%$ decline in FVC and the percentage with no decline in percentage predicted FVC, were compared in a pooled analysis at serial time-points. Secondary outcomes included change in 6-min walk distance (6MWD) and/or death, change in dyspnoea as measured with the University of California San Diego Shortness of Breath Questionnaire (UCSD SOBQ), and progression-free survival. In the combined study populations, the effect on FVC was in clear favour of the pirfenidone arm at 12 months, as well as at the time of study completion in the respective trials. Those who received 1 year of treatment were $>40 \%$ less likely to reach the threshold of a $10 \%$ fall in FVC or death and $38 \%$ less likely to progress at all, compared with those on placebo. There was also a distinct separation of the effect on FVC as early as 3 months, which was maintained at each subsequent time-point. These findings are similar to the treatment effect demonstrated in the ASCEND patient population, supporting the relevance of the results of this study.

With regard to secondary end-points in the combined population, a decline in $6 \mathrm{MWD}$ of $\geqslant 50 \mathrm{~m}$ or death, and progression-free survival were both mitigated significantly by pirfenidone at 1 year and at study completion. In the combined study, fewer patients in the pirfenidone group experienced a clinically relevant fall in their UCSD SOBQ scores at 12 months, compared with the placebo arm, a finding not demonstrated in the individual clinical trial populations.

It is often tempting to consider a therapeutic intervention to be more effective in certain subgroups. Is the treatment benefit present in the elderly or in patients with mild disease, for example? Interestingly, in the pooled study population, Noble et al. [1] report a treatment effect in favour of pirfenidone across all age groups, including the elderly (>75 years), and all stages of disease severity, including mild disease (FVC $>80 \%$ predicted and diffusing capacity of the lung for carbon monoxide $>50 \%$ predicted), irrespective of smoking status or sex.

NoBLE et al. [1] also report safety findings similar to those in each of the individual trials. Gastrointestinal, skin and liver function test anomalies constituted the majority of issues, but rarely led to treatment cessation. Interestingly, patients with greater physiological impairment at baseline had a greater chance of experiencing more severe adverse events, although this relationship was not different between the intervention and placebo arms.

Do these combined analyses provide further insights beyond those demonstrated by the individual clinical trials? NoBLE et al. [1] argue that pooled data analysis is a widely used method to develop a more accurate estimation of the magnitude of treatment effect, including the effect on outcomes that occurred with a low frequency in the individual studies. They propose that the increased patient population increases the power allowing assessment of the treatment effect in subpopulations of interest. Indeed, pooled data for the TOMORROW and INPULSIS studies of nintedanib have also been undertaken for assessment of mortality [14]. In this larger patient cohort of 1231 patients, there was a numerical trend towards lower mortality in the patients treated with nintedanib. No doubt, further insights with regard to safety and efficacy could be made.

NoBLE et al. [1] acknowledge the limitations of their pooled analysis and inferential statistics. Efforts are made to overcome the differences in trial duration. The subtle variations in study populations are not to be ignored: eligibility criteria for ASCEND were modified from the CAPACITY studies, resulting in a more homogeneous IPF population augmented to enrol patients with a higher risk of disease progression. On balance, these minor inconsistencies are unlikely to contribute significant bias, particularly as the pooled results are similar to that of the ASCEND study.

For any pooled data cohort, the question should be asked if this is the most appropriate method of analysis, considering these acknowledged differences in study population and duration (72 weeks in CAPACITY versus 52 weeks in ASCEND). Would meta-analysis be a more robust approach, in order to overcome some of the limitations of inference? Meta-analysis is an appropriate method to approach such populations including combined data sets. In the recent meta-analysis of thrombolysis for pulmonary 
embolism, for example, a mortality benefit was demonstrated that no single trial was adequately powered to detect [15].

What, then, can meta-analyses of pirfenidone in IPF tell us? In 2015, ArAvenA et al. [16] showed with meta-analysis that pirfenidone improved progression-free survival, all-cause and disease-specific mortality, and parameters associated with disease progression. This included the two Japanese pirfenidone studies, along with the ASCEND and CAPACITY trials. The relative risk of severe adverse events was no different between placebo and intervention groups, although milder treatment-specific side-effects were prevalent. Tolerance data were also evaluated in the meta-analysis of IPF and hypersensitivity pneumonitis patients by JIANG et al. [17], demonstrating increased risks of mild gastrointestinal, dermatological and neurological complaints. A subsequent network meta-analysis, looking at the effectiveness of various treatments for IPF, including pirfenidone and nintedanib, showed that only these two therapies were of significant benefit [18]. The limitations of this form of analysis were the indirect comparison of treatments not formally assessed in head-to-head trials and the combination of different end-points to gauge efficacy.

Certainly, the findings of the pooled analysis by NoBle et al. [1] are in alignment with these recent meta-analyses. Additional important insights, however, can be gleaned with regard to the overall treatment impact, the effect within certain subgroups and various safety outcomes.

Noble et al. [1] conclude that these pooled data are stable and robust estimates of the magnitude of the treatment effect of pirfenidone in IPF. They show a treatment benefit across several subgroups and a homogenous safety profile. So who are the patients that should be treated with pirfenidone or nintedanib in the "real world"? Patients and their families often bring expectations of receiving specific treatment, even at their initial specialist consultation. Should clinicians be limiting their prescribing to those patients who fulfil trial entry criteria (and indeed, which criteria should be followed)? Or rather, should a more pragmatic approach be taken, with extrapolation outside of these strict parameters to our clinic patients [19]? For the clinician, it is reassuring to see a consistent treatment effect across population subgroups. It will be interesting to see how such data are able to inform prescription. Should all IPF patients with mild disease receive anti-fibrotic medications? This is a question that will no doubt be debated, and influenced by local regulatory authorities and guidelines, as well as patient preference.

The analysis by NoBLE et al. [1], along with the ASCEND and INPULSIS trials, certainly advances our understanding of how best to manage patients with IPF. Real-world data gathered from national registries and post-marketing surveillance will help to address some of the uncertainties [20]. Ongoing and future clinical trials will necessarily include background antifibrotic therapy [10,21]. We have come a long way in a short time, but there is still much to be learnt about this complex disease. In an era of increasingly personalised medicine and with an evolving understanding of specific biological mechanisms, it is likely that combination therapy will be the way of the future. Patient-driven outcomes, including cough, dyspnoea and quality of life, will also require focused attention if we are to make further inroads in this disease [22]. In the real word, physicians are still facing a series of unanswered questions. The story is by no means over.

\section{References}

1 Noble PW, Albera C, Bradford WZ, et al. Pirfenidone for idiopathic pulmonary fibrosis: analysis of pooled data from three multinational phase 3 trials. Eur Respir J 2016; 47: 243-253.

2 Noble PW, Albera C, Bradford WZ, et al. Pirfenidone in patients with idiopathic pulmonary fibrosis (CAPACITY): two randomised trials. Lancet 2011; 377: 1760-1769.

3 Iyer SN, Wild JS, Schiedt MJ, et al. Dietary intake of pirfenidone ameliorates bleomycin-induced lung fibrosis in hamsters. J Lab Clin Med 1995; 125: 779-785.

4 Oku H, Shimizu T, Kawabata T, et al. Antifibrotic action of pirfenidone and prednisolone: different effects on pulmonary cytokines and growth factors in bleomycin-induced murine pulmonary fibrosis. Eur J Pharmacol 2008; 590: 400-408.

5 Schelegle ES, Mansoor JK, Giri S. Pirfenidone attenuates bleomycin-induced changes in pulmonary functions in hamsters. Proc Soc Exp Biol Med 1997; 216: 392-397.

6 Raghu G, Johnson WC, Lockhart D, et al. Treatment of idiopathic pulmonary fibrosis with a new antifibrotic agent, pirfenidone: results of a prospective, open-label Phase II study. Am J Respir Crit Care Med 1999; 159: 1061-1069.

7 Azuma A, Nukiwa T, Tsuboi E, et al. Double-blind, placebo-controlled trial of pirfenidone in patients with idiopathic pulmonary fibrosis. Am J Respir Crit Care Med 2005; 171: 1040-1047.

8 Taniguchi H, Ebina M, Kondoh Y, et al. Pirfenidone in idiopathic pulmonary fibrosis. Eur Respir J 2010; 35: 821-829.

9 Noble PW, Albera C, Bradford WZ, et al. Pirfenidone in patients with idiopathic pulmonary fibrosis (CAPACITY): two randomised trials. Lancet 2011; 377: 1760-1769.

10 Collard HR, Bradford WZ, Cottin V, et al. A new era in idiopathic pulmonary fibrosis: considerations for future clinical trials. Eur Respir J 2015; 46: 243-249.

11 King TE Jr, Bradford WZ, Castro-Bernardini S, et al. A phase 3 trial of pirfenidone in patients with idiopathic pulmonary fibrosis. N Engl J Med 2014; 370: 2083-2092.

12 Richeldi L, du Bois RM, Raghu G, et al. Efficacy and safety of nintedanib in idiopathic pulmonary fibrosis. $N$ Engl J Med 2014; 370: 2071-2082. 
13 Raghu G, Rochwerg B, Zhang Y, et al. An official ATS/ERS/JRS/ALAT clinical practice guideline: treatment of idiopathic pulmonary fibrosis. An update of the 2011 clinical practice guideline. Am J Respir Crit Care Med 2015; 192: e3-e19.

14 Kirsten AM, et al. Pooled analysis of mortality data form the TOMORROW and INPULSIS trials of nintedanib in idiopathic pulmonary fibrosis (IPF). Pneumologie 2015; 69: P246.

15 Chatterjee S, Chakraborty A, Weinberg I, et al. Thrombolysis for pulmonary embolism and risk of all-cause mortality, major bleeding, and intracranial hemorrhage: a meta-analysis. JAMA 2014; 311: 2414-2421.

16 Aravena C, Labarca G, Venegas C, et al. Pirfenidone for idiopathic pulmonary fibrosis: a systematic review and meta-analysis. PLoS One 2015; 10: e0136160.

17 Jiang C, Huang $\mathrm{H}$, Liu J, et al. Adverse events of pirfenidone for the treatment of pulmonary fibrosis: a meta-analysis of randomized controlled trials. PLoS One 2012; 7: e47024.

18 Loveman E, Copley VR, Colquitt JL, et al. The effectiveness and cost-effectiveness of treatments for idiopathic pulmonary fibrosis: systematic review, network meta-analysis and health economic evaluation. BMC Pharmacol Toxicol 2014; 15: 63.

19 Moodley Y, Corte T, Richeldi L, et al. Do all patients with idiopathic pulmonary fibrosis warrant a trial of therapeutic intervention? A pro-con perspective. Respirology 2015; 20: 389-394.

20 Ryerson CJ, Corte TJ, Collard HR, et al. A global registry for idiopathic pulmonary fibrosis: the time is now. Eur Respir J 2014; 44: 273-276.

21 Wuyts WA, Antoniou KM, Borensztajn K, et al. Combination therapy: the future of management for idiopathic pulmonary fibrosis? Lancet Respir Med 2014; 2: 933-942.

22 Russell AM, Sprangers MA, Wibberley S, et al. The need for patient-centred clinical research in idiopathic pulmonary fibrosis. BMC Med 2015; 13: 240. 